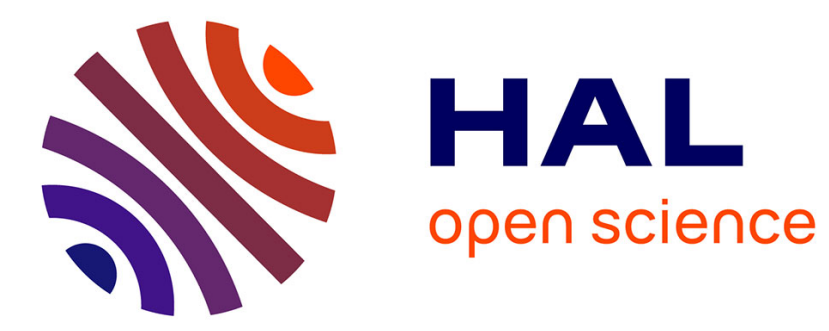

\title{
Remarques sur les interactions magnétiques
}

Louis Néel

\section{To cite this version:}

Louis Néel. Remarques sur les interactions magnétiques. Journal de Physique et le Radium, 1962, 23

(8-9), pp.449-452. 10.1051/jphysrad:01962002308-9044900 . hal-02887456

\section{HAL Id: hal-02887456 \\ https://hal.science/hal-02887456}

Submitted on 2 Jul 2020

HAL is a multi-disciplinary open access archive for the deposit and dissemination of scientific research documents, whether they are published or not. The documents may come from teaching and research institutions in France or abroad, or from public or private research centers.
L'archive ouverte pluridisciplinaire HAL, est destinée au dépôt et à la diffusion de documents scientifiques de niveau recherche, publiés ou non, émanant des établissements d'enseignement et de recherche français ou étrangers, des laboratoires publics ou privés. 


\title{
LE JOURNAL DE PHYSIQUE
}

ET

\section{I.E RADIUM}

\section{COLLOQUE DE MAGNÉTISME}

Organisé à Orsay et Saclay

les 27 et 28 avril 1962 par la

Société françatse de Physique

Section de Physique des Solides

\section{REMARQUES SUR LES INTERACTIONS MAGNÉTIQUES}

Par L. NÉEL,

Faculté des Sciences, Grenoble.

\begin{abstract}
Résumé. - Après un bref rappel historique, l'auteur passe en revue les méthodes permettant de déterminer l'énergie des interactions magnétiques : les unes fournissent l'interaction moyenne entre deux atomes, les autres la moyenne du produit de l'interaction par le carré de la distance des deux atomes intéressés. Il expose notamment un exemple appartenant à la deuxième catégorie : une méthode précise de mesure de l'énergie des parois de Bloch.

Ces deux catégories de méthodes prennent aujourd'hui une importance accrue, au moment où de nouvelles et importantes théories montrent que la portée des interactions est plus grande qu'on ne l'avait cru jusqu'ici.
\end{abstract}

\begin{abstract}
After a short historical introduction, the author reviews the methods for determining the energy of magnetic interactions : some give the average interaction of two atoms, others the average of the product of the interaction energy by the square of the distance between the atoms. A special case of the second group is discussed : a precise method of measuring Bloch wall energies.

These two groups of methods take on more significance, today, now new and important theories show that the range of interactions is greater than had been supposed previously.
\end{abstract}

Introduction. - Dès le moment où en 1906 dans sa théorie phénoménologique du ferromagnétisme $P$. Weiss représenta les interactions entre les porteurs de moment par un champ magnétique,

$$
H_{\mathrm{m}}=N J \text {, }
$$

il reconnut le caractère fictif de ce champ et l'impossibilité d'attribuer les interactions à des couplages dipolaires magnétiques. Dans la suite après la théorie de Debye et les expériences fixant l'ordre de grandeur des moments électriques dipolaires P. Weiss précisa l'idée de l'origine électrostatique du champ moléculaiie en supposant qu'un même atome possédait des moments magnétique et électrique couplés. Sous une forme plus subtile et grâce au jeu du principe d'exclusion de Pauli Heisenberg montra ensuite en 1928 par quel mécanisme les forces électriques pouvaient intervenir dans un couplage magnétique. Un tel couplage lié à des échanges d'électrons et à l'empiètement des fonctions d'onde ne pouvait prendre une valeur notable qu'entre atomes proches voisins et le caractère essentiellement local des interactions magnétiques parut dès lors assez bien établi pour que plusieurs auteurs estimassent légitime de déduire des résultats expérimentaux des courbes donnant l'intensité de ces interactions en fonction de la distance des atomes proches voisins.

Cependant en 1934 dans un mémoire qui attira assez peu l'attention Kramers découvrit la possi- 
bilité d'interactions dites de superéchange entre deux atomes séparés par un atome non magnétique, par exemple un atome d'oxygène. En 1948, L. Néel montra que c'était à de telles interactions qu'il fallait attribuer le ferrimagnétisme et l'antiferromagnétisme des ferrites et des oxydes.

Entre temps et dans le cas des métaux de la fin de la première série de transition échouaient les multiples efforts pour préciser théoriquement les valeurs des interactions d'échange : actuellement il ne semblent pas qu'elles. puissent atteindre l'ordre de grandeur voulu.

Une idée nouvelle est alors apparue celle du rôle des électrons de conduction $4 s$ et de leur polarisation par le moment magnétique de la couche $3 d$ : il en résulte un couplage par l'intermédiaire de la couche $4 s$. Ces conceptions se sont beaucoup précisées au cours de ces dernières années grâce notamment aux travaux de Friedel et de ses collaborateurs. Elles jettent un jour nouveau et encourageant sur des problèmes qui apparaissaient quelque peu dans une impasse.

Détermination expérimentale des interactions. La diversité des interprétations théoriques relatives aux interactions magnétiques donne un prix encore plus grand aux comparaisons de la théorie avec l'expérience. Quelles sont donc les méthodes dont nous disposons pour déterminer ces interactions ? Il semble que nous en possédions deux grandes catégories : les unes donnent l'énergie d'aimantation c'est-à-dire une quantité telle que

$$
E=\sum_{i, j} w_{i j}
$$

les autres donnent le coefficient de l'énergie d'une onde de spin, c'est-à-dire une quantité telle que

$$
A=\sum_{i, j} a_{i j}^{2} w_{i j}
$$

où $w_{i j}$ représente l'énergie de couplage de deux atomes $i$ et $j$ et $a_{i j}$ leur distance mutuelle, les deux sommes étant étendues à tous les couples possibles d'atomes du système. Lorsqu'il existe seulement des interactions entre atomes proches voisins à une distance $a$ connue $E$ et $A$ donnent les mêmes renseignements, mais si il n'en est pas ainsi nous obtenons deux données indépendantes dont la connaissance présente le plus grand intérêt.

Chaleur spécifique. - En principe l'anomalie de chaleur spécifique fournit directement $E$ par la mesure de l'aire comprise entre la courbe représentant la variation thermique de la chaleur spécifique du corps ferromagnétique et une courbe de référence représentant la chaleur spécifique de la même substance supposée privée de son ferromagnétisme.

Malheureusement les mesures de chaleur spécifique sont difficiles surtout à haute température et en l'espèce il faut toujours monter assez loin car les anomalies de chaleur spécifique d'un ferromagnétique se poursuivent bien au-dessus du point de Curie à la suite de la persistance à haute température d'un ordre à courte distance.

D'autre part, le tracé de la courbe de référence présente de très grandes incertitudes soit que l'on prenne comme référence la chaleur spécifique d'un corps de propriétés physiques voisines (le cuivre, dans le cas du nickel, par exemple) soit que l'on essaye d'extrapoler vers les basses températures la partie de la courbe de chaleur spécifique du ferromagnétique étudié correspondant à des températures largement supérieures à la température de Curie.

Il y a à tenir compte enfin pour les métaux d'un terme de chaleur spécifique électronique des électrons de la bande $3 d$ pratiquement indissociable $d u$ terme magnétique et présent à toute température.

En fait, la méthode n'a guère été appliquée qu'au nickel, avec une précision assez faible : peutêtre $10 \%$ [1]. Elle a fourni des résultats en accord avec la méthode suivante.

Point de Curie paramagnétique vrai $\theta_{\mathrm{p}}$. D'après la théorie du champ moléculaire, l'énergie magnétique $-\frac{1}{2} N J_{s}^{2}$, peut se calculer connaissant l'aimantation spontanée $J_{s}$ et le coefficient $N$ du champ moléculaire que l'on peut calculer d'après la relation $N=\theta_{\mathfrak{p}} / C$, où $C$ est la constante de Curie.

Malheureusement $\theta_{p}$ correspond à l'intersection avec l'axe des températures de l'asymptote à la courbe représentant la variation thermique de l'inverse de la susceptibilité magnétique, au-dessus du point de Curie. Cette asymptote est très difficile à déterminer car nous ne disposons jamais que des mesures correspondant à un intervalle très restreint de températures, avec au voisinage du point de Curie ferromagnétique une courbure assez forte et il est bien possible que dans ces conditions l'asymptote soit encore assez éloignée de la courbe. La littérature se borne à donner un point de Curie paramagnétique apparent $\theta_{\mathfrak{p}}^{\prime}$ correspondant à l'extrapolation d'une région à peu près rectiligne de la courbe $\left(\frac{1}{\chi}, T\right)$ et il ne serait pas étonnant que $\theta_{\mathrm{p}}^{\prime}$ soit de 10 à $20 \%$ inférieur à $\theta_{\mathrm{p}}$.

Point de Curie ferromagnétique $\theta_{\mathrm{f}}$. - On peut aussi songer à déterminer $\theta_{\mathrm{f}}$ et à en déduire $\theta_{\mathrm{p}}$, au moyen d'une correction calculée à l'aide d'une théorie statistique convenable du ferromagnétisme du genre de celles qui ont été développées par Domb et ses collaborateurs. Malheureusement ces théories sont extrêmement difficiles et comportent généralement des restrictions variées : couplages du type Ising, spin faible, interactions réduites aux plus proches voisins, etc... Les calculs ne s'appli- 
quent certainement pas à des interactions de signe alterné, du type Friedel, s'étendant à relativenent grande distance. Dans l'état actuel de ces théories, il ne paraît donc pas possible d'espérer déduire $\theta_{\mathbf{p}}$ de $\theta_{\mathrm{f}}$ à mieux de cinq ou même $10 \%$ près.

Loi d 'approche thermique à la saturation absolue. - La théorie de Bloch qui repose sur l'emploi des ondes thermiques de spin permet de déduire $A$ du coefficient $b$ de la loi d'approche à la saturation

$$
J_{\mathrm{s}}=J_{\mathrm{s} 0}\left(1-b T^{3 / 2}\right) \text {. }
$$

Un moment donné, la détermination de $b$ avait paru pratiquement impossible à cause de la présence, dans le développement de $J_{\mathrm{s}}$ en fonction des puissances de $T$, d'un terme en $T^{7 / 4}$ susceptible d'interférer sérieusement [2] avec le terme en $T^{3 / 2}$. En fait, il n'en est rien, comme l'a montré J.F. Dyson, le terme suivant étant en $T^{5 / 2}$. La loi (1) est valable dans un intervalle de température suffisamment étendu $\left(0<T<0,4 \theta_{\mathrm{f}}\right)$ pour permettre une détermination de $A$ précise à 5 ou $10 \%$. La méthode s'applique aisément à tous les ferromagnétiques.

Torsion de l'aimantation spontanée dans les lames minces. - Une méthode originale due à Methfessel, Middlehoek et Thomas [3] permet de déterminer $A$, dans un métal uniaxe magnétiquement. On envoie dans une lame très mince de ce métal, parallèle à l'axe, un courant électrique également parallèle à l'axe qui crée des champs magnétiques perpendiculaires à l'axe, mais de signes contraires sur les deux faces opposées de la lame. Ces champs magnétiques dispersent en éventail l'aimantation spontanée : l'ouverture de l'éventail, d'autant plus grande que la lame est plus épaisse et que $A$ est plus petit, se déduit de la diminution de la valeur moyenne de la projection sur l'axe de l'aimantation spontanée ; on augmente la sensibilité en mesurant la modulation à la fréquence $2 f$ de l'aimantation moyenne produite par un courant électrique de fréquence $f$ et on augmente la précision en faisant des mesures pour différentes valeurs d'un champ additionnel $H_{x}$ parallèle à l'axe, ce qui permet de faire varier l'anisotropie apparente. Pour obtenir de bons résultats, il faut disposer de lames dont l'épaisseur est de l'ordre de grandeur de l'épaisseur des parois de Bloch, $1000 \AA$ pour fixer les idées. De telles lames sont obtenues par évaporation ou par projection cathodique : il n'est pas certain que la structure de lames aussi minces soit celle du métal massif et divers effets de surface peuvent y prendre une importance relativement grande.

Quoi qu'il en soit, la méthode a été appliquée à un film de permalloy et a fourni une valeur de $A$, précise à $10 \%$, concordant avec la valeur fournie par la loi d'approche thermique à la saturation absolue.
Ondes de spin excitées par micro-ondes. - On peut aussi exciter en haute fréquence des ondes de spin (harmoniques d'ordre impair) dans une lame mince et en déduire $A$. La méthode, qui a été appliquée au permalloy [4] et plus récemment au cobalt, quoique plus difficilement, s'applique à des lames qui sont presque aussi minces que celles qui sont employées dans la méthode précédente et est soumise par conséquent aux mêmes objections.

Énergie $\gamma$ des parois de Bloch. - Cette énergie est proportionnelle à la racine carrée de $A$ et à celle de l'énergie d'anisotropie, relativement facile à mesurer par ailleurs. Différentes méthodes de mesure de $\gamma$ ont été proposées. L'une d'elles, basée sur des calculs de L. Néel [5] et sur la mesure de l'épaisseur moyenne des domaines élémentaires en forme de feuillets parallèles qui se forment dans une lame monocristalline de fer, taillée parallèlement à un axe binaire [6], ne fournit que des résultats peu précis (30\% près), probablement parce qu'elle est trop sensible aux défauts et aux tensions internes.

Une autre, mise en œuvre avec un plus grand succès [7], consiste à aimanter longitudinalement un fil cylindrique, puis, au moyen d'une brusque inversion du champ magnétique, à créer une paroi cylindrique qui, partant de la surface du fil, tend à diminuer de diamètre sous l'influence de la tension superficielle. Cette méthode ne s'applique qu'à des substances ferromagnétiques isotropes et nécessite des fils dont la fabrication est quelquefois difficile. En fait, elle n'a été appliquée qu'à des fils de ferronickel $(65 \% \mathrm{Ni}, 35 \% \mathrm{Fe})$ tendus et a fourni une valeur de $A$, avec une précision estimée à $20 \%$.

Récemment une méthode qui paraît plus précise a été proposée par L. Néel [8]. Elle utilise des lames minces ferromagnétiques monocristallines à faces parallèles d'une épaisseur de quelques dixièmes de millimètres et dont les propriétés doivent ainsi être très voisines de celles de la substance massive. On crée dans cette lame des parois de Bloch à $180^{\circ}$, perpendiculaires aux faces de la lame, dont on observe les traces sur les deux faces par la méthode de Bitter. On envoie ensuite dans la lame qui doit être conductrice de l'électricité, un courant électrique perpendiculaire à la direction des aimantations spontanées des deux domaines élémentaires adjacents à la paroi étudiée. Ce courant produit un champ magnétique dont les valeurs sont de signes contraires sur les deux faces de la lame. Les deux traces de la paroi tendent à se déplacer en sens contraires : un équilibre s'établit alors entre la pression magnétique et les forces de tension superficielle qui s'opposent à l'augmentation de surface de la paroi. On déduit l'énergie superficielle des valeurs de la densité de courant et du déplacement des traces. Il veut veiller à tenir compte des variations de l'énergie de paroi avec l'orientation.

Cette méthode a été appliquée avec beaucoup de 
succès par P. Brissonneau et R. Aléonard [9] sur divers échantillons d'épaisseurs variées de fersilicium à $3 \%$ de $\mathrm{Si}$ : les auteurs ont obtenu une énergie de $1,52 \mathrm{erg} / \mathrm{cm}^{2}$, avec une précision meilleure que $5 \%$.

Comparaison des valeurs de $E$ et de $A$. - Il est bien évident que cette comparaison systématique pour un grand nombre de corps ferromagnétiques présenterait beaucoup d'intérêt. Malheureusement nous ne disposons pas actuellement des données nécessaires.

Dans le cas du fer-silicium à $3 \% \mathrm{Si}$, une évaluation encore provisoire semble montrer qu'avec l'hypothèse d'une seule espèce de voisins magnétiquement actifs, séparés par la même distance $a$, cette distance $a$ est plus voisine de $2,86 \AA$, distance des 6 seconds voisins situés suivant les axes quaternaires, que de $2,50 \AA$, distance des 8 premiers voisins situés suivant les axes ternaires. Ce résultat s'accorde avec une suggestion ancienne de L. Néel [10] selon laquelle ce seraient les seconds voisins $d u$ fer qui seraient magnétiquement actifs, les premiers correspondant à des interactions sensiblement nulles.

Cependant, si, comme le suggèrent les travaux théoriques récents, il faut introduire des interactions à plus longue distance, avec des signes positifs ou négatifs, la valeur expérimentale de $a$ donnée par $\sqrt{A / E}$ prend une signification plus complexe dont l'interprétation n'est pas immédiate.

Signalons pour terminer que nous avons supposé implicitement que les interactions magnétiques ne dépendaient pas de la température : ce n'est pas du tout certain. Beaucoup de faits suggèrent [10], [11] la possibilité de variations thermiques du coefficient de champ moléculaire telles que sa valeur $N$ au point de Curie diffère de 5 à $10 \%$ de sa valeur $N_{0}$ au zéro absolu : cela complique encore la discussion.

\section{BIBLIOGRAPHIE}

[1] Lapp (E.), Ann. Physique, 1929, 12, 442.

[2] Scharroth (M. A.), Proc. Phil. Soc., London, 1954, A 67, 33.

[3] Methfessel (S.), Middelhoek (S.) et Thomas (H.), Proc. Int. Conf. on Magnetism and Cristallography, Kyoto, Sept. 1961.

[4] Seavey (M. H.) et Tannenwald (P. E.), Phys. Rev. Letters, 1958, 1, 168.

[5] Néel (L.), J. Physique Rad., 1944, 5, 241.

[6] Martins (D. H.), Proc. Phys. Soc., 1957, 70 B, 77.
[7] Bean (C. P.) et De Blois (R. W.), Magnetic properties of Metals and Alloys, p. 18 (American Society for Metals, Cleveland, Ohio, 1959).

[8] NéEL (L.), C. R. Acad. Sc., 1962, 254, 2891.

[9] Aléonard (R.) et Brissonneau (P.), C. R. Acad. Sc., 1962, 254, 2934.

[10] Néel (L.), Réunion Internationale de Magnétisme, Strasbourg, 1939 (tome II, Ferromagnétisme, Coll. Scient. Inst. Int. Coop. Intellectuelle, Paris, 1940, pp. 65-104).

[11] Néel (L.), Ann. Physique, 1937, 8, 237. 